\title{
Oscillating on Borrowed Time: Diffusible Signals from Immortalized Suprachiasmatic Nucleus Cells Regulate Circadian Rhythmicity in Cultured Fibroblasts
}

\author{
Gregg Allen, ${ }^{1}$ Jodie Rappe, ${ }^{2}$ David J. Earnest, ${ }^{1}$ and Vincent M. Cassone ${ }^{2}$ \\ ${ }^{1}$ Department of Human Anatomy and Medical Neurobiology, Texas A\&M University Health Science Center, College of \\ Medicine, College Station, Texas 77843-1114, and '2Department of Biology, Texas A\&M University, College Station, Texas \\ 77843-3258
}

The capacity to generate circadian rhythms endogenously and to confer this rhythmicity to other cells was compared in immortalized cells derived from the suprachiasmatic nucleus (SCN) and a fibroblast line to differentiate SCN pacemaker properties from the oscillatory behavior of non-clock tissues. Only SCN2.2 cells were capable of endogenously generating circadian rhythms in 2-deoxyglucose uptake and Per gene expression. Similar to SCN function in vivo, SCN2.2 cells imposed rhythms of metabolic activity and Per gene expression on cocultured NIH/3T3 fibroblasts via a diffusible signal. The conferred rhythms in NIH/3T3 cells were phase delayed by 4-12 hr relative to SCN2.2 circadian patterns, thus resembling the phase relationship between SCN and peripheral tissue rhythms in vivo. Sustained metabolic rhythmicity in NIH/3T3 cells was dependent on continued exposure to SCN2.2specific outputs. In response to a serum shock the NIH/3T3 fibroblasts exhibited recurrent oscillations in clock gene expression, but not in metabolic activity. These molecular rhythms in serum-shocked fibroblasts cycled in a phase relationship similar to that observed in the SCN in vivo; peak Per1 and Per2 mRNA expression preceded the rhythmic maxima in Cry1 and Cry 2 mRNA levels by $4 \mathrm{hr}$. Despite these clock gene oscillations the serum-shocked NIH/3T3 cells failed to drive circadian rhythms of Per1 and Per2 expression in cocultures of untreated fibroblasts, suggesting that expression and circadian regulation of the Per and Cry genes are not sufficient to confer pacemaker function. Therefore, SCN-specific outputs are necessary to drive circadian rhythms of metabolic activity, and these output signals are not a direct product of clock gene oscillations.

Key words: circadian pacemaker; Clock; oscillation; suprachiasmatic nucleus; glucose use; Per1; Per2; Cry1; Cry2; coculture
In multicellular animals, circadian rhythmicity is a universal property of biochemical and physiological processes and is controlled predominantly by nervous and neuroendocrine structures. Destruction of specific clock tissues abolishes or severely alters the expression of overt rhythmicity, and, where possible, transplantation of these structures restores circadian rhythmicity to the organism (Rusak and Zucker, 1979). In mammals the circadian clock has been localized to a single structure: the hypothalamic suprachiasmatic nucleus (SCN) (Klein et al., 1991). SCN ablation eliminates circadian patterns of behavioral activity, endocrine output, and many biochemical processes throughout the body (Turek, 1985), and transplantation of the SCN anlagen restores circadian behavioral activity in SCN-lesioned hosts (Ralph et al., 1990). Furthermore, SCN cells express circadian rhythms of electrical, metabolic, and biochemical activity in vivo and in vitro (Schwartz, 1991; Gillette, 1997; Earnest et al., 1999b). Identification of the molecular elements responsible for these profound pacemaking properties of the SCN circadian oscillator is there-

\footnotetext{
Received May 9, 2001; revised July 31, 2001; accepted Aug. 2, 2001.

This study was supported by National Institutes of Health (NIH) Program Project Grant P01 NS39546 (D.J.E. and V.M.C.) and NIH Grant NS35822 (V.M.C.). G.C.A., D.J.E., and V.M.C. are NHTs. We thank Rodney Walline for excellent technical assistance and Deb Bell-Pedersen and Susan Golden for their valuable comments on this manuscript.

Correspondence should be addressed to Dr. David J. Earnest, Texas A\&M University Health Science Center, Department of Human Anatomy and Medical Neurobiology, 238 Reynolds Medical Building, College Station, TX 77843-1114. E-mail: dearnest@tamu.edu.

Copyright (C) 2001 Society for Neuroscience $0270-6474 / 01 / 217937-07 \$ 15.00 / 0$
}

fore of critical importance for understanding the circadian timekeeping mechanism in mammals.

Recent advances in the genetic dissection of the circadian clockwork in mammals have identified genes for which the products oscillate with a circadian periodicity and interact within an interlocked transcriptional/translational feedback loop that is thought to constitute the central clock mechanism (Shearman et al., 2000b). Mutation or knock-out of at least four of these genes, tau in golden hamsters and Clock (Clk), Cryptochrome1 (Cry1), and Cryptochrome2 (Cry2) in mice, alters or abolishes the circadian rhythm of activity (Ralph and Menaker, 1988; Vitaterna et al., 1994; van der Horst et al., 1999). Three orthologs of the Drosophila period gene, Per1, Per2, and Per3, also have been cloned and characterized in mammals (Shearman et al., 1997; Zylka et al., 1998), although their specific functions in the circadian clockwork have not been revealed fully by studies examining the effects of mutations in individual Per genes on behavioral rhythmicity (Zheng et al., 1999, 2001; Shearman et al., 2000a; Bae et al., 2001). Intriguingly, these "clock genes" are expressed and oscillate in many peripheral tissues as well as in the SCN (Shearman et al., 1997; Zylka et al., 1998). Further, circadian oscillations in clock gene expression can be induced in fibroblast cell lines by serum shock and/or pulses of corticosteroid hormones at pharmacological dosages (Balsalobre et al., 1998, 2000; Duffield et al., 2000). These observations have challenged the presumption that the core genetic components and their rhythmic regulation must be localized uniquely in the SCN. Collectively, these findings raise several important questions: Is the peripheral expression of 
clock gene rhythmicity indicative of pacemaker function in these tissues? If not, what differences in these molecular oscillations distinguish the pacemaker properties of the SCN from peripheral oscillators?

To approach these issues, we examined molecular and physiological indices in several cell lines that exhibit circadian pacemaker function and/or endogenous oscillatory activity. Our immortalized line of rat SCN cells (SCN2.2) provided a central focus, because these cells retain the capacity of the SCN in situ both to generate circadian rhythms in their own physiological processes and to restore behavioral rhythmicity to the entire animal when transplanted into SCN-lesioned hosts (Earnest et al., 1999a,b). As noted earlier, the capacity to oscillate is not a unique property of the $\mathrm{SCN}$, because rhythmic expression of various clock and clock-controlled genes for which the transcripts also show oscillating abundance in peripheral tissues in vivo has been observed in cultures of the Rat-1 and NIH/3T3 fibroblast lines after serum shock treatment (Balsalobre et al., 1998; Akashi and Nishida, 2000). On the basis of these findings, we conducted a series of experiments to address the following questions: Can diff usible output signals from SCN2.2 cells drive both molecular and physiological rhythms in cocultured NIH/3T3 fibroblasts? Is continued exposure to these circadian signals necessary, or is a brief exposure from SCN2.2 cells sufficient to "kick-start" and sustain NIH/3T3 rhythmicity? Can a serum shock induce NIH/ $3 \mathrm{~T} 3$ fibroblasts to express molecular and physiological rhythms similar to those imposed by SCN-specific outputs? Do serumshocked NIH/3T3 fibroblasts exhibit circadian pacemaker properties by driving molecular rhythms in cocultured cells?

\section{MATERIALS AND METHODS}

Propagation of cell lines and general culture conditions. SCN2.2 and $\mathrm{NIH} / 3 \mathrm{~T} 3$ fibroblast lines were propagated without antibiotics on culture dishes ( $60 \mathrm{~mm}$; Corning, Corning, NY) and maintained at $37^{\circ} \mathrm{C}$ and $5 \%$ $\mathrm{CO}_{2}$ in MEM containing 10\% FBS, $3000 \mu \mathrm{g} / \mathrm{ml}$ glucose, and $292 \mu \mathrm{g} / \mathrm{ml}$ L-glutamine. During cell propagation the medium was changed at $48 \mathrm{hr}$ intervals, and the cultures were split every $2-3 \mathrm{~d}$.

Experiment 1: Can SCN2.2 cells confer metabolic and molecular rhythmicity onto NIH/3T3 fibroblasts? SCN2.2 and NIH/3T3 cells derived from a single passage were expanded in parallel on multiple companion plates (Falcon, Oxnard, CA). Colonies of NIH/3T3 cells were established on cell-impermeable inserts $(23 \mathrm{~mm}$; pore size, $1 \mu \mathrm{m})$ and cocultured with companion wells $(35 \mathrm{~mm})$ containing either SCN2.2 cells or similar NIH/3T3 cultures. Beginning $36 \mathrm{hr}$ after plating the cultures $(n=$ 4 for SCN2.2-NIH/3T3 cocultures; $n=6$ for NIH/3T3-NIH/3T3 cocultures) were collected at $4 \mathrm{hr}$ intervals for $52 \mathrm{hr}$ to determine 2-deoxyglucose (2-DG) uptake and Per2 mRNA expression in each coculture compartment. This analysis was performed on four to six sets of companion inserts and wells at each time point. Cells were harvested with Trizol reagent (Life Technologies, Gaithersburg, MD) to extract soluble protein and total cellular RNA. Extracted cellular RNA for a given coculture subtype at each time point was pooled into a single sample, and aliquots were analyzed for expression of rat Per2 (rPer2; SCN2.2), mouse Per2 (mPer2; NIH/3T3), and species-specific $\beta$-actin mRNA by ribonuclease protection assay.

To analyze Per1 transcription in SCN2.2 and NIH/3T3 cells, we transfected a reporter vector (pGL3, Promega, Madison, WI) containing a $3 \mathrm{~kb}$ fragment of the Per1 promoter fused to the firefly luciferase (luc) gene (kindly provided by Dr. Charles Weitz, Harvard University) and a downstream cassette encoding the blasticidin resistance gene (Invitrogen, San Diego, CA) into both cell types via liposome-mediated introduction (Lipofectamine Plus, Life Technologies). Selection of stable integrants was conducted by passing the cultures $\sim 24 \mathrm{hr}$ after transfection and then subjecting the cells to growth in the presence of blasticidin (3-10 $\mu \mathrm{g} / \mathrm{ml}$ ) for $5 \mathrm{~d}$. SCN2.2 and NIH/3T3 cells expressing the Per1/luc transgene were plated on companion wells and inserts, respectively. After $36 \mathrm{hr}$ in coculture the cell lysates were harvested separately from each compartment at $4 \mathrm{hr}$ intervals for $52 \mathrm{hr}$. Enzyme activity in lysates was analyzed by the Luciferase Assay System (Promega). Bioluminescent activity associated with Perl/luc transgene expression was measured in counts per second with a Packard TopCount scintillation counter (Meriden, CT); the values were normalized for sample protein content.

Experiment 2: Is continued exposure to SCN2.2 outputs necessary to sustain NIH/3T3 rhythmicity? NIH/3T3 fibroblasts were plated on companion wells and maintained alone or were cocultured with SCN2.2 cells on inserts. After SCN2.2 and NIH/3T3 cells were cocultured together for $36 \mathrm{hr}$, the inserts were removed and both cell types were maintained separately in isolation. Then $24 \mathrm{hr}$ later 2-DG uptake was determined at $4 \mathrm{hr}$ intervals for $52 \mathrm{hr}$. Cells were harvested by lysis in phosphate buffer for the extraction of total protein and scintillation counting.

Experiment 3: Can a serum shock induce both metabolic and molecular rhythmicity in NIH/3T3 fibroblasts? Using identical methods to those described by Balsalobre and colleagues (1998), we exposed confluent cultures of NIH/3T3 cells on T75 flasks to medium containing $50 \%$ adult horse serum for $2 \mathrm{hr}$ and then maintained them under serum-free conditions. Uptake of 2-DG and expression of mouse-specific clock gene mRNAs were analyzed in cultures collected at $4 \mathrm{hr}$ intervals for $52 \mathrm{hr}$. Cells were harvested with Trizol reagent to extract soluble protein and total cellular RNA. Extracted cellular RNA at each time point was analyzed for $\mathrm{mPer} 1, \mathrm{mPer} 2, \mathrm{~m} C r y 1, \mathrm{~m} C r y 2$, and mouse-specific $\beta$-actin mRNA levels by ribonuclease protection assay.

Experiment 4: Do serum-shocked NIH/3T3 fibroblasts exhibit circadian pacemaker properties by driving molecular rhythms in cocultured cells? $\mathrm{NIH} / 3 \mathrm{~T} 3$ cells derived from a single passage were expanded on multiple companion plates (Costar, Pleasanton, CA) in normal growth medium (DMEM supplemented with $10 \%$ FBS, $3500 \mu \mathrm{g} / \mathrm{ml}$ glucose, and 292 $\mu \mathrm{g} / \mathrm{ml}$ L-glutamine). Before experimentation, transwell inserts $(75 \mathrm{~mm})$ and companion wells $(100 \mathrm{~mm})$ containing colonies of NIH/3T3 cells were established and cultured separately. At confluence only NIH/3T3 cultures on companion wells were subjected to replacement of normal growth medium and exposed to medium containing 50\% adult horse serum for $2 \mathrm{hr}$. After serum shock treatment the medium was exchanged with serum-free DMEM (supplemented with glucose and L-glutamine), and then these $\mathrm{NIH} / 3 \mathrm{~T} 3$ cultures on companion wells were cocultured with inserts containing untreated NIH/3T3 cells in which normal growth medium had been replaced to establish similar serum-free conditions. Cells were harvested from each coculture compartment at $4 \mathrm{hr}$ intervals for $52 \mathrm{hr}$ with RNeasy (Qiagen, Chatsworth, CA). At each time point total cellular RNA from individual plates and their corresponding inserts was analyzed for $\mathrm{mPer} 1$ and $\mathrm{mPer} 2 \mathrm{mRNA}$ levels by ribonuclease protection assay.

Measurement of 2-DG uptake. SCN2.2 and NIH/3T3 cells were assayed for uptake of 2-DG via methods described previously (Earnest et al., 1999b). Confluent cultures were incubated for $1 \mathrm{hr}$ with ${ }^{14} \mathrm{C}$-labeled 2-DG (0.1 mCi/ml; American Radiological, St. Louis, MO) and rinsed twice with Dulbecco's PBS (without calcium or magnesium). Cells then were harvested by lysis either with Trizol reagent (for extraction of soluble protein and total RNA as in Experiments 1 and 3) or in phosphate buffer. Aliquots of cell lysates $(100-200 \mu \mathrm{l})$ were placed in scintillation vials in triplicate and then counted on a Beckman scintillation counter. Determinations of 2-DG uptake were normalized for sample protein content as measured by the bicinchoninic acid method (Pierce, Rockford, IL).

Ribonuclease protection assays. Radiolabeled $\left(\alpha{ }^{-32} \mathrm{P}-\mathrm{CTP} ; 800 \mathrm{Ci} / \mathrm{mM}\right)$ antisense RNA probes were generated for $\mathrm{rPer} 2(\mathrm{mRNA}$ position +287 to +165$), \mathrm{mPer} 1(+761$ to +340$), \mathrm{mPer} 2(+489$ to +9$), \mathrm{mCry} 1(+1793$ to +1074$)$, mCry2 $(+1696$ to +1040$)$, rat $\beta$-actin $(+2779$ to +2682$)$, and mouse $\beta$-actin $(+989$ to +739$)$ by in vitro transcription of linearized templates. RNA probes were gel fractionated, and size-appropriate bands were extracted. Mouse-specific probes were used to examine expression of Per, Cry, and $\beta$-actin mRNAs in NIH/3T3 fibroblasts, and rat-specific probes were used for parallel analysis in SCN2.2 cells.

After isolation of whole-cell RNA the samples were treated with RNase-free DNase (Promega) to digest genomic DNA and were stored at $-80^{\circ} \mathrm{C}$. Ribonuclease protection assays were performed with the RPAIII kit (Ambion, Austin, TX). Individual RNA samples from NIH/3T3 fibroblasts were subjected to multiprobe hybridizations (experimental probe and mouse-specific $\beta$-actin). For SCN2.2 cells separate hybridization reactions with experimental and control probes were performed on each RNA sample because of close similarity in the size of the protected fragments for rat-specific Per 2 and $\beta$-actin mRNAs. Single and multiprobe hybridizations were performed with $10 \mu \mathrm{g}$ of total RNA and high-specific-activity probes (Per and Cry, $\approx 2-5 \times 10^{8} \mathrm{cpm} / \mu \mathrm{g} ; \beta$-actin, $\approx 2 \times 10^{6} \mathrm{cpm} / \mu \mathrm{g}$ ) for $20 \mathrm{hr}$ at $56^{\circ} \mathrm{C}$. The nonhybridized cRNA probes 


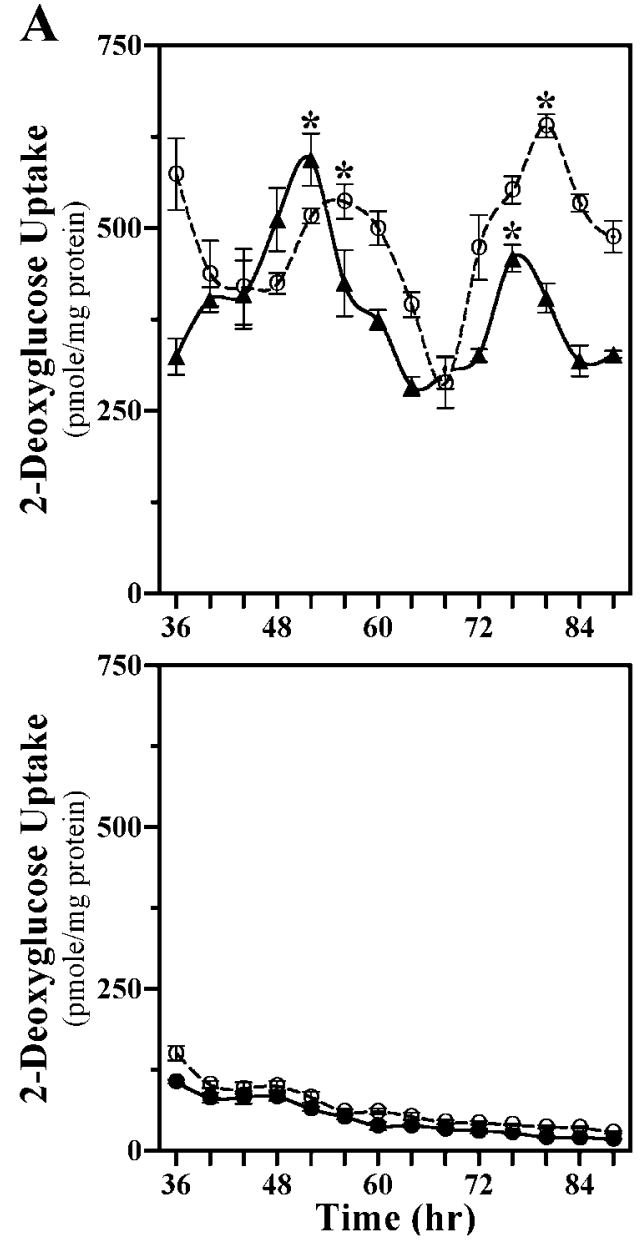

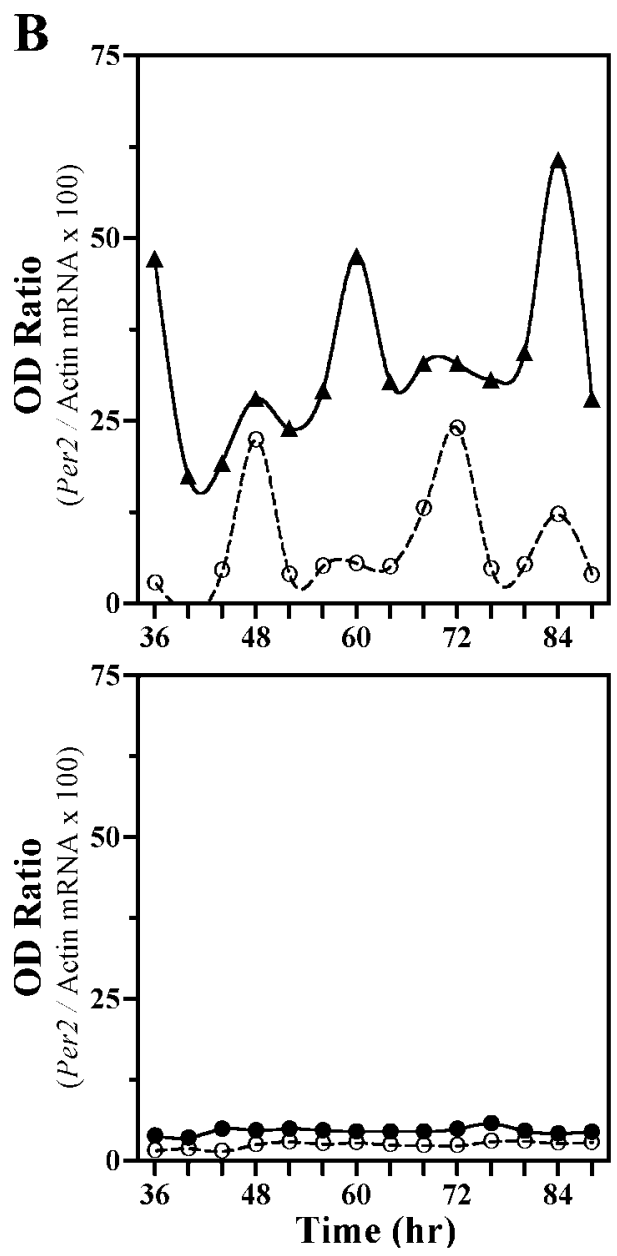

Figure 1. SCN2.2 are distinguished by the capacity to drive metabolic and molecular rhythmicity in cocultured NIH/3T3 fibroblasts. Shown are temporal patterns of 2-deoxyglucose (2-DG) uptake $(A)$ and Per 2 expression (B) in cocultures (top) containing SCN2.2 cells on wells $(n=4$; solid line, $\mathbf{\Delta})$ and $\mathrm{NIH} / 3 \mathrm{~T} 3$ fibroblasts on inserts $(n=4$; dashed line, $\bigcirc)$ and in cocultures (bottom) composed of NIH/3T3 cells on both wells $(n=6$; solid line,, ) and inserts $(n=6$; dashed line, $\bigcirc)$. The symbols denote determinations of 2-DG uptake (mean \pm SEM) and optical density $(O D)$ ratios of $P e r 2 / \beta$-actin mRNA signal at $4 \mathrm{hr}$ intervals. With the exception of Figure 4, determinations in this and subsequent figures are plotted as a function of time such that time 0 denotes when cells located on companion wells and inserts were cocultured together first. Asterisks indicate sampling intervals during which peak values for 2-DG uptake were significantly greater $(p<0.05)$ than those observed during the preceding or succeeding minima. and mRNAs were digested with $2.5 \mathrm{U} / \mathrm{ml}$ RNase A and $100 \mathrm{U} / \mathrm{ml}$ RNase $\mathrm{T} 1$ at $35^{\circ} \mathrm{C}$ for $30 \mathrm{~min}$. Protected cRNA:mRNA fragments were fractionated on $5 \%$ acrylamide $/ 8 \mathrm{M}$ urea gels. Radiolabeled bands were visualized from dried gels by film autoradiography. Optical density measurements for size-appropriate Per1, Per2, Cry1, Cry2, and $\beta$-actin mRNAs were obtained with a Bio-Rad PhosphorImager (GS-525; Hercules, CA) and Molecular Analyst software. For each sample the optical density of the Per1, Per2, Cry 1, or Cry 2 band was normalized to that for $\beta$-actin to control for variation in sample RNA content and allow for comparison of relative differences in Per and Cry mRNA expression. Optical density comparisons between $\beta$-actin and each experimental mRNA were derived from the same gel for NIH/3T3 fibroblasts and from separate gels for SCN2.2 cells.

Statistical analysis. Time-dependent fluctuations were identified by one-way ANOVA; paired comparisons between determinations at specific time points were analyzed post hoc for statistical differences with the Newman-Keuls sequential range test.

\section{RESULTS}

\section{Experiment 1: Can SCN2.2 cells confer metabolic and molecular rhythmicity onto NIH/3T3 fibroblasts?}

SCN2.2 cells and NIH/3T3 fibroblasts were maintained in a coculture environment and assessed separately for evidence of rhythmicity in their uptake of 2-DG, a well documented marker of circadian metabolic activity (Newman and Hospod, 1986; Schwartz, 1991), and expression of putative clock genes Per1 and Per2. Consistent with our earlier findings (Earnest et al., 1999b), SCN2.2 cells expressed a circadian rhythm in 2-DG uptake for two cycles when cocultured with NIH/3T3 fibroblasts (Fig. 1A, top). SCN2.2 cells also exhibited circadian rhythmicity in Per2 mRNA expression (Fig. 1B, top), with maximal levels occurring $\sim 8 \mathrm{hr}$ after the peak in 2-DG uptake. When cocultured with SCN2.2 cells, NIH/3T3 fibroblasts displayed analogous circadian rhythms of 2-DG uptake and Per2 expression. In addition, the profile of Perl gene regulation was analyzed in a separate set of cocultures containing SCN2.2 and NIH3T3 cells that express the Per1 promoter linked to a luciferase reporter gene. Similar to 2-DG uptake and Per2 expression, Per1-driven luciferase bioluminescence was clearly rhythmic in both SCN2.2 and NIH/3T3 cells (Fig. 2, top). In SCN2.2-NIH/3T3 cocultures, peak levels of 2-DG uptake and Per gene expression were approximately twofold greater than the corresponding minimum for rhythms in both cell types. However, the phase of the conferred rhythmicity in $\mathrm{NIH} / 3 \mathrm{~T} 3$ cells was delayed by $4 \mathrm{hr}$ for the rhythm in 2-DG uptake and by $12 \mathrm{hr}$ for the oscillations in Per gene expression relative to the circadian patterns observed in cocultured SCN2.2 cells. For the oscillations in $\mathrm{Per}$ gene expression this phase relationship between SCN2.2 and NIH/3T3 rhythms is similar to that reported between the SCN and peripheral tissues in vivo (LopezMolina et al., 1997; Shearman et al., 1997; Zylka et al., 1998) and in vitro (Yamazaki et al., 2000). Without the influence of SCN2.2 cells those cultures containing only NIH/3T3 cells showed no evidence of circadian variation in 2-DG uptake, Per2 mRNA levels (see Fig. 1A,B, bottom), and Perl-driven luciferase bioluminescence (Fig. 2, bottom) when cocultured on inserts and wells 


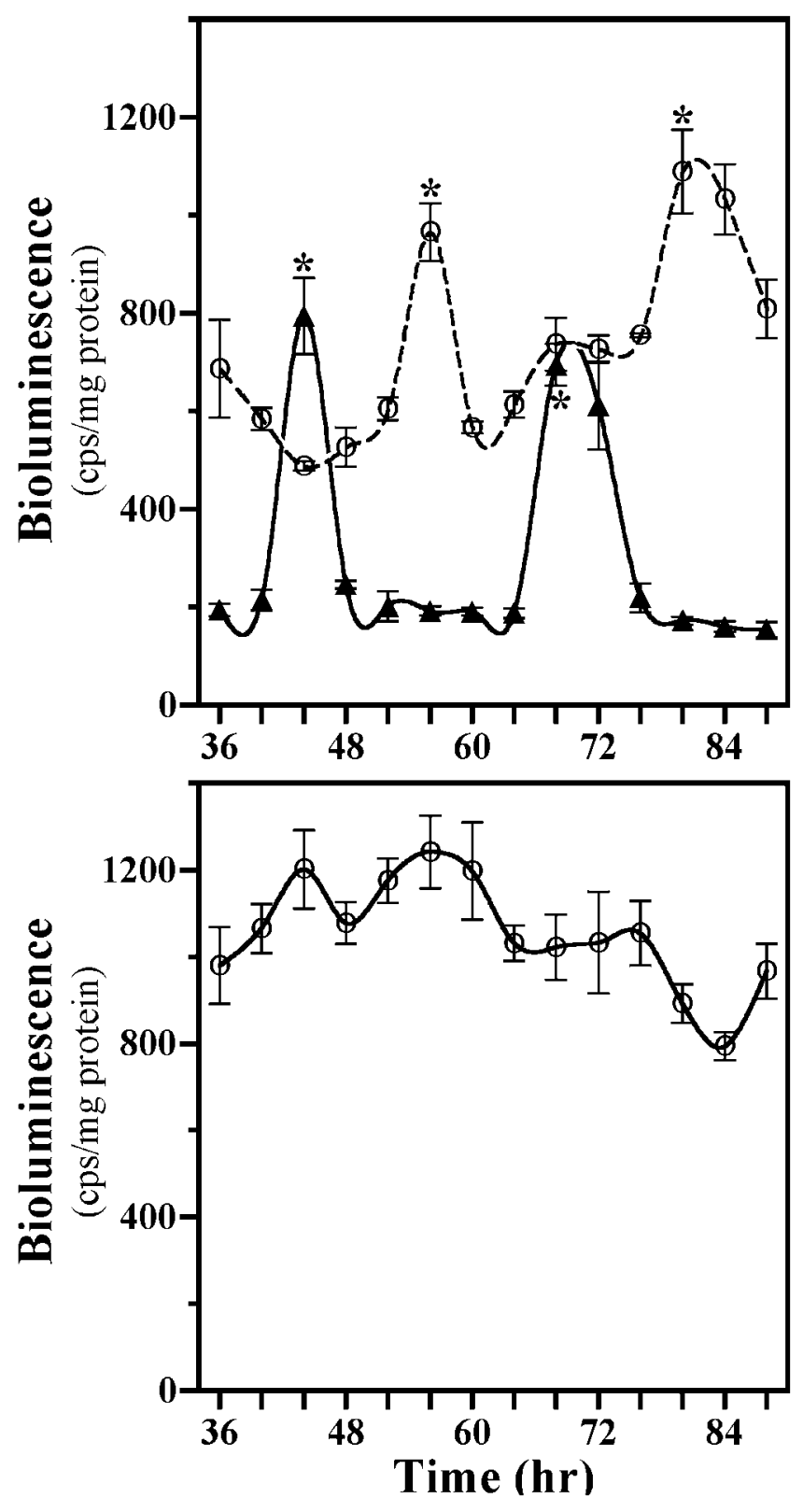

Figure 2. Profiles of Per1/luc transgene expression in cocultures (top) containing SCN2.2 cells on wells $(n=3$; solid line, $\mathbf{\Delta})$ and NIH/3T3 fibroblasts on inserts $(n=3$; dashed line, $O)$ and in NIH/3T3 cells cultured alone (bottom) on wells $(n=3$; solid line, $\bigcirc)$. The symbols denote determinations of Per1-driven luciferase bioluminescence (mean \pm SEM) at $4 \mathrm{hr}$ intervals. Asterisks indicate sampling intervals during which peak values for Per1-driven luciferase bioluminescence were significantly greater $(p<0.05)$ than those observed during the preceding or succeeding minima.

or maintained in isolation. In addition to the absence of rhythmicity, NIH/3T3-NIH/3T3 cocultures were distinguished by low levels of 2-DG uptake and Per2 mRNA expression in comparison with those observed in SCN2.2 cells or other NIH/3T3 cells exposed to the influence of SCN2.2 signals. Collectively, these results demonstrate that only SCN2.2 cells are endogenously capable of generating rhythms in metabolic activity and Per gene expression, whereas NIH/3T3 cells require the presence of SCN2.2-specific outputs to express physiological as well as molecular rhythmicity.

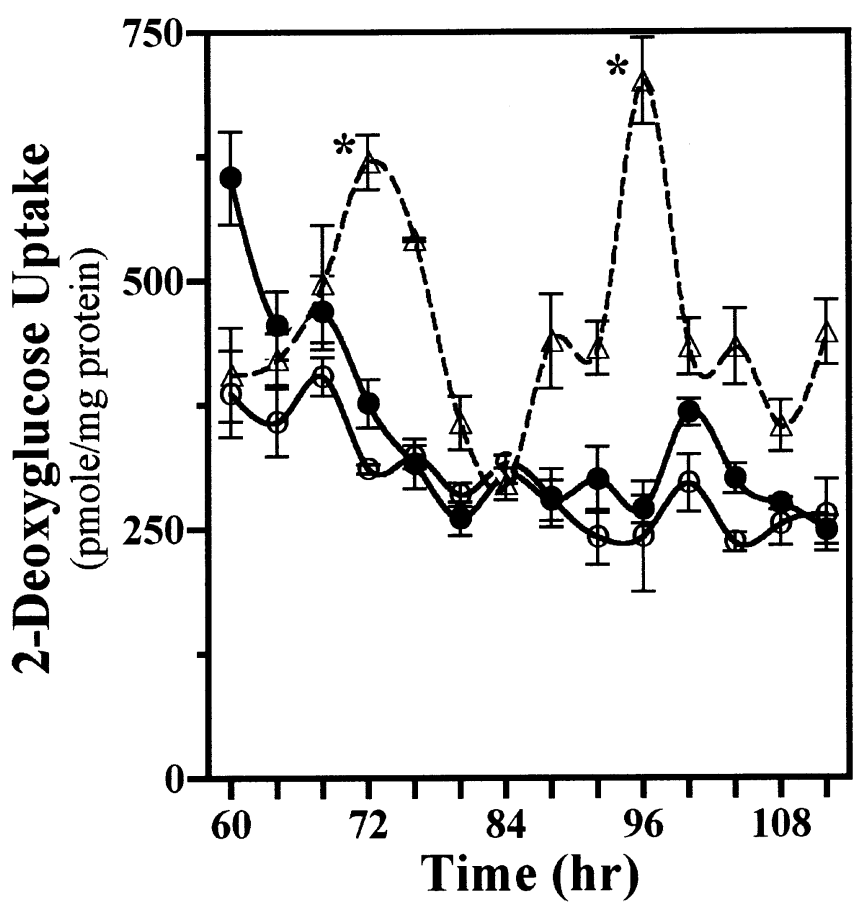

Figure 3. Conferred rhythmicity in NIH/3T3 cells persists only in the presence of SCN2.2 cells. Shown is a "kick-start" analysis of 2-DG uptake in SCN2.2 cells on inserts $(n=3$; dashed line, $\triangle)$ and NIH/3T3 fibroblasts on wells $(n=3$; solid line, - that were cocultured together for only $24 \mathrm{hr}$ and maintained separately thereafter. $\mathrm{NIH} / 3 \mathrm{~T} 3$ fibroblasts cultured alone on wells $(n=3$; solid line, $\bigcirc)$ are shown for comparison. The symbols denote mean \pm SEM determinations of 2-DG uptake at $4 \mathrm{hr}$ intervals. Asterisks indicate sampling intervals during which peak values were significantly greater $(p<0.05)$ than those observed during the preceding or succeeding minima.

\section{Experiment 2: Is continued exposure to SCN2.2 outputs necessary to sustain NIH/3T3 rhythmicity?}

Mixed cocultures were evaluated next for the persistence of rhythmic 2-DG uptake after the removal of SCN2.2 cells to determine whether a brief exposure to some diffusible signal from these cells simply kick-starts NIH/3T3 oscillations similar to those induced by a serum shock (Balsalobre et al., 1998; Akashi and Nishida, 2000; Duffield et al., 2000). After their coculture and subsequent isolation from each other, SCN2.2 cells on inserts expressed circadian fluctuations in their 2-DG uptake for 2 cycles, whereas NIH/3T3 cells on companion wells failed to show continued oscillations in metabolic activity (Fig. 3). Beyond the initial elevation the 2-DG uptake in NIH/3T3 fibroblasts with previous coculture exposure to SCN2.2 cells showed little variation from basal levels observed in NIH/3T3 cells that had been cultured alone. These findings suggest that SCN2.2 cells impart circadian rhythmicity on NIH/ 3T3 fibroblasts via a diffusible output signal for which the continuous and/or fluctuating presence are/is required for sustained metabolic rhythmicity and for phase control of this oscillation.

\section{Experiment 3: Can a serum shock induce both metabolic and molecular rhythmicity in NIH/3T3 fibroblasts?}

Because serum shock induces molecular oscillations in Rat-1 fibroblasts (Balsalobre et al., 1998), effects of this stimulus on 2-DG uptake and clock gene expression in NIH/3T3 cells on culture flasks were examined to provide comparison with the oscillations conferred by diffusible signals from cocultured 


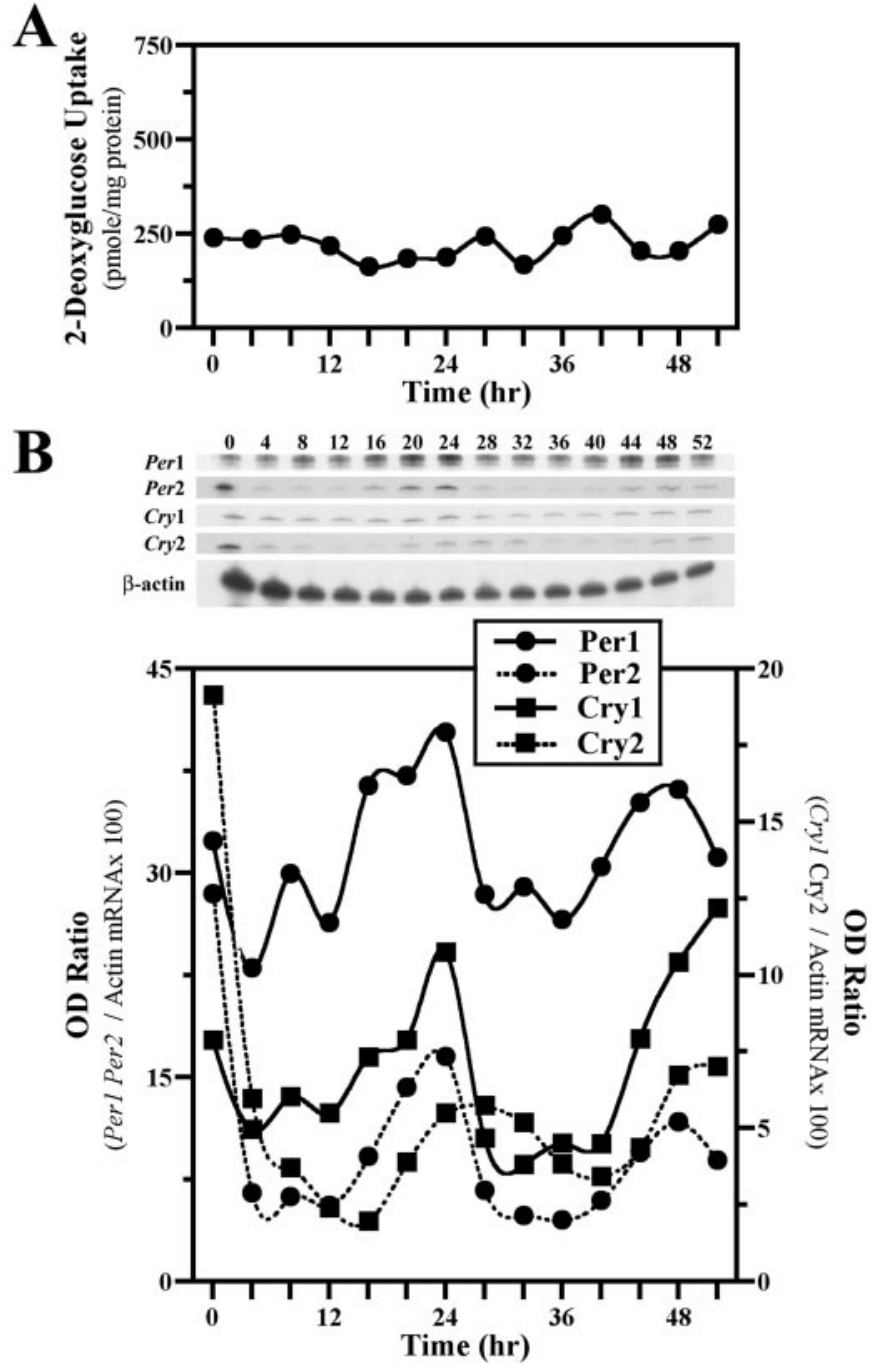

Figure 4. Serum shock induces molecular, but not metabolic, rhythmicity in NIH/3T3 fibroblasts. Shown are temporal patterns of 2-DG uptake $(A)$ and Clock gene expression $(B)$ in cultures of NIH/3T3 cells after a $2 \mathrm{hr}$ exposure to a $50 \%$ serum shock. Determinations of $2-\mathrm{DG}$ uptake and OD ratios of $\mathrm{mPer} 1, \mathrm{mPer} 2, \mathrm{mCry} 1$, or $\mathrm{mCry} 2 / \beta$-actin mRNA signal at $4 \mathrm{hr}$ intervals are plotted such that time 0 coincides with the conclusion of the serum shock treatment.

SCN2.2 cells. Metabolic activity in serum-shocked NIH/3T3 fibroblasts was arrhythmic (Fig. 4A), with 2-DG uptake remaining mainly invariable at levels comparable to those observed in the preceding experiment for solitary NIH/3T3 cultures. In contrast, serum shock induced rhythms of clock gene expression in $\mathrm{NIH} /$ 3 T3 cells (Fig. 4B). Per1, Per2, Cry1, and Cry2 gene expression was elevated immediately after exposure to the $2 \mathrm{hr}$ serum shock $(0 \mathrm{hr})$; thereafter, mRNA abundance profiles of these genes continued to oscillate for two cycles. These molecular rhythms were robust and characterized by a two- to threefold elevation from nadir to peak levels of Per1, Per2, Cry1, and Cry2 mRNA. Paralog comparisons of clock gene oscillations in serum-shocked NIH/3T3 cells revealed contemporary cycling of Per1 and Per2 mRNAs and of Cry1 and Cry 2 mRNAs. However, the rhythmic expression profiles for the Cry genes were phase delayed during the second cycle by $4 \mathrm{hr}$ relative to those for the Per genes. This phase relationship between the rhythms in Per and Cry gene expression for serum-shocked NIH/3T3 cells is comparable to

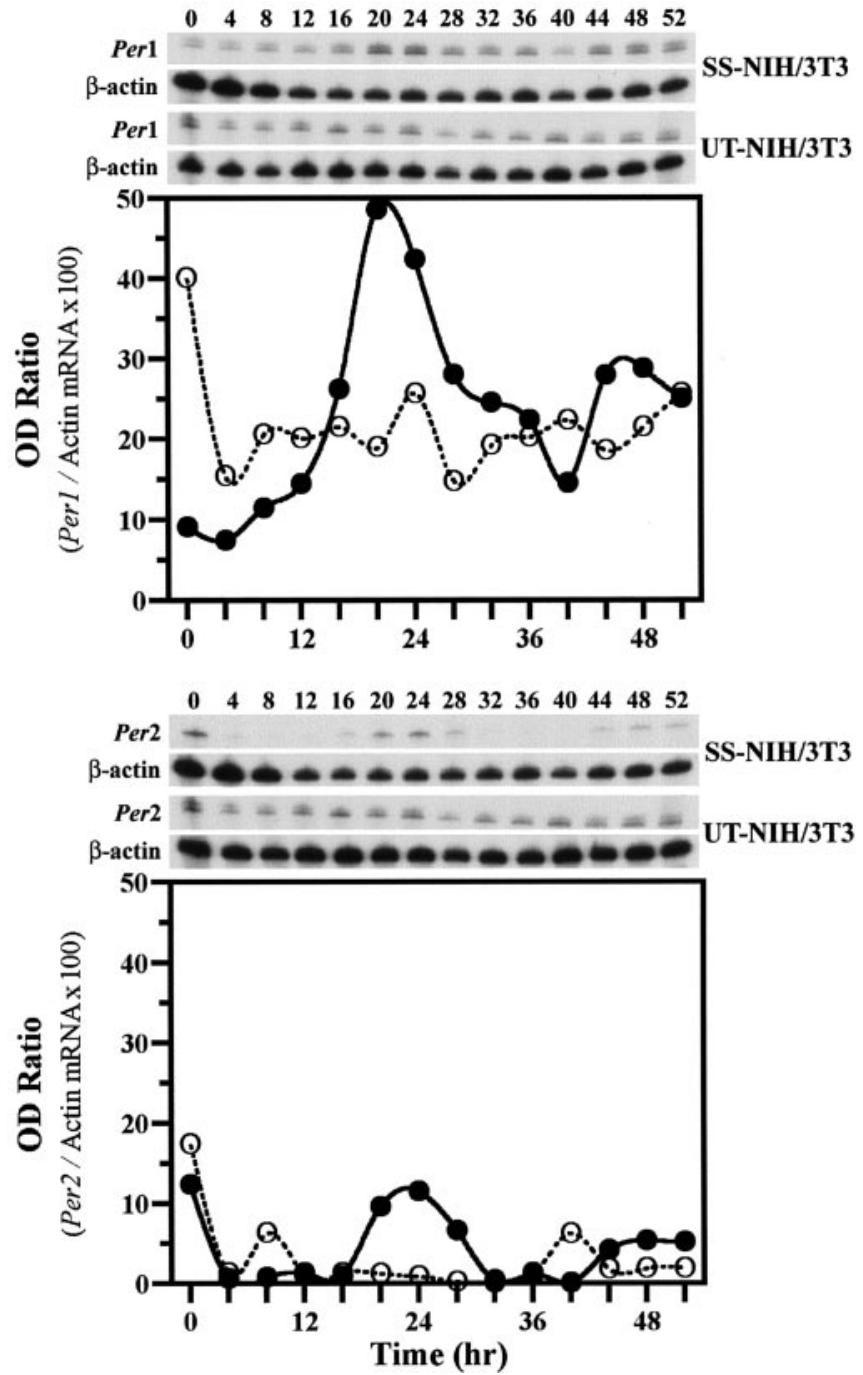

Figure 5. Serum-shocked NIH/3T3 fibroblasts express molecular oscillations but cannot drive rhythmicity in cocultured cells. Shown are temporal patterns of mPer1 (top) and mPer2 (bottom) mRNA expression in $\mathrm{NIH} / 3 \mathrm{~T} 3-\mathrm{NIH} / 3 \mathrm{~T} 3$ cocultures. NIH/3T3 cells on wells $(\mathrm{SS}-\mathrm{NIH} / 3 \mathrm{~T} 3$; solid line, -) were exposed to a $2 \mathrm{hr}$ serum shock $(50 \%)$ and then cocultured with untreated NIH/3T3 fibroblasts on inserts (UT-NIH/3T3; dashed line, $\bigcirc)$. The symbols denote OD ratios of $\mathrm{mPer} 1$ or $\mathrm{mPer} 2 / \beta$-actin mRNA signal at $4 \mathrm{hr}$ intervals.

that reported for the SCN in vivo (Shearman et al., 2000a) and for Rat-1 fibroblasts in vitro (Yagita et al., 2001).

\section{Experiment 4: Do serum-shocked NIH/3T3 fibroblasts exhibit circadian pacemaker properties by driving molecular rhythms in cocultured cells?}

Because the observed oscillations in their expression of the Per and Cry genes resemble SCN molecular rhythms in vivo (Shearman et al., 2000a), serum-shocked NIH/3T3 cells were examined in a coculture environment for evidence of pacemaker properties analogous to SCN2.2 cells. Consistent with the preceding experiment, exposure to a $2 \mathrm{hr}$ serum shock elicited synchronous cycling of Perl and Per2 mRNA levels in NIH/3T3 fibroblasts maintained on companion wells (Fig. 5). In these serum-shocked fibroblasts the peak mRNA levels for the Per1 and Per2 oscillations were initially 5 - to 10 -fold greater than the corresponding minimum, with considerable damping of rhythm amplitude during the second cycle. Unlike the serum-shocked constituents of 
these cocultures, untreated NIH/3T3 cells on inserts showed no overt signs of circadian fluctuations in Per1 or Per2 gene expression. Aside from an initial elevation at the beginning of the sampling interval, Per1 and Per2 mRNA levels in untreated NIH/ 3 T3 fibroblasts remained mainly within the range of the minimal values observed in serum-shocked cells.

\section{DISCUSSION}

The present experiments demonstrate that immortalized cells derived from the rat SCN are capable of (1) endogenously generating circadian rhythms in both 2-DG uptake and Per gene expression and (2) conferring this metabolic and molecular rhythmicity to the NIH/3T3 fibroblast line. The specific mechanism by which SCN2.2 cells drive circadian rhythms in NIH/3T3 cells is not known at this point. However, it is likely that a diffusible signal released from SCN2.2 cells imposes rhythmicity on NIH/ 3 T3 fibroblasts, because the two cell types were separated by a semi-permeable membrane preventing physical contact. Furthermore, the continued presence of this SCN-communicated signal is necessary for the generation of metabolic rhythmicity in NIH/ $3 \mathrm{~T} 3$ cells. After coculture together for $36 \mathrm{hr}$ and subsequent separation the SCN2.2 cells continued to show a circadian pattern of metabolic activity, whereas NIH/3T3 fibroblasts were marked by an immediate loss of rhythmicity (Fig. 2). This finding indicates that SCN2.2 cells rhythmically release some paracrine signals responsible for the circadian regulation of metabolism in downstream cells. Although molecular oscillations were not examined in this specific experiment, rhythmic expression of clock genes in NIH/3T3 cells similarly may depend on the continuous influence of circadian output signals from SCN2.2 cells unless molecular and physiological rhythms are uncoupled in the fibroblast model.

Possible involvement of a diffusible factor in the SCN pacemaker regulation of circadian rhythms has distinct precedent. Transplantation of fetal hypothalamic tissue and/or cells into the third ventricle of SCN-lesioned rodents restores circadian rhythms frequently with few, if any, neural connections between the graft and host brain (Lehman et al., 1987; Ralph et al., 1990; Aguilar-Roblero et al., 1994). The most convincing evidence for SCN pacemaker communication via a humoral output is provided by transplantation experiments demonstrating that $\mathrm{SCN}$ cell grafts, even when isolated in semi-permeable capsules, confer circadian rhythmicity to the behavior of SCN-lesioned hosts (Silver et al., 1996). Thus, diffusible signals may be sufficient to drive at least some circadian rhythms in surrounding tissues via paracrine mechanisms. Identification of these paracrine factors will be critical in elucidating how circadian outputs from the SCN pacemaker regulate downstream tissues and cells (Silver et al., 1996; LeSauter and Silver, 1998).

Perhaps the most interesting observation in the present study is that, despite their expression of all of the known "clock genes" (Per1, Per2, Cry1, Cry2, BMAL1, and Clock), NIH/3T3 cells do not generate circadian rhythms by themselves without some form of experimental intervention. Therefore, mere expression of these clock genes is not sufficient alone to produce rhythmicity. Even when circadian oscillations in Per1, Per2, Cry1, and Cry2 mRNA levels are induced in NIH/3T3 cells by serum shock (Fig. $3 B)$, this rhythmic gene expression is still not sufficient to generate rhythmicity in physiological metabolism (Fig. $3 A$ ). Thus, signals expressed by SCN2.2 cells and presumably the SCN, which are not Per1, Per2, Cry1, or Cry2, are necessary for endogenous rhythmicity and for pacemaker function (i.e., the ability to confer rhythmicity). It is important to emphasize that the canonical clock genes and their rhythmicity may still be necessary, albeit not sufficient, for overt rhythmicity. Systematic knock-out of these genes and/or their gene products from SCN2.2 cells will be necessary to determine this.

There is little doubt that mammalian orthologs of the Drosophila "clock genes" are involved in the regulation of mammalian circadian rhythms (Reppert and Weaver, 2000; Shearman et al., 2000b). Mutations in tau, now known to encode a casein kinase homologous to Drosophila double-time (dbt) (Lowrey et al., 2000), or the positive element Clock (Clk) in addition to knock-out of the two cryptochromes Cry 1 and Cry 2 change circadian period or abolish circadian rhythms altogether (Reppert and Weaver, 2000). Further, in vitro analyses indicate that "negative elements" such as the mammalian cryptochromes interact with the Per genes to inhibit activity of "positive elements" Clk and Bmall, which dimerize and activate E-box elements on promoter regions of these and other genes (Gekakis et al., 1998; Kume et al., 1999). Finally, recent evidence suggests that core components of this molecular feedback loop regulate downstream clock-controlled genes, such as arginine vasopressin (AVP) and albumin D binding protein (DBP), via interaction with E-box regulatory sequences (Gekakis et al., 1998; Jin et al., 1999; Ripperger et al., 2000).

Although this transcription-translation feedback mechanism is clearly the guiding model for the clockworks in Drosophila and the filamentous fungus Neurospora crassa (Young, 1998), it is not clear whether this feedback loop is sufficient to explain the present data. The components of the clock are present in pacemaker configuration only within SCN2.2 cells, which are capable of driving both rhythmic gene expression and metabolism intrinsically and in cells downstream of the clock. It is noteworthy that the conferred rhythms in NIH/3T3 fibroblasts are phase delayed by 4-12 hr relative to those in SCN2.2 cells (Fig. 1). Importantly, this circadian phase difference suggests that SCN2.2 cells do not induce or impose directly the NIH/3T3 metabolic and molecular oscillations in a manner analogous to a masking effect unless SCN2.2-communicated signals require 4-12 hr to diffuse and act on NIH/3T3 cells. The phase relationship between SCN2.2 and $\mathrm{NIH} / 3 \mathrm{~T} 3$ rhythms is also consistent with that observed between the SCN and peripheral tissues in vivo (Lopez-Molina et al., 1997; Shearman et al., 1997; Zylka et al., 1998).

The observed functional differences between SCN2.2 and $\mathrm{NIH} / 3 \mathrm{~T} 3$ cells are important in relation to the application of fibroblast lines in a number of recent investigations to study the molecular components of the circadian clock mechanism in mammals. In a thoughtful and entertaining rejoinder to the idea that the mammalian retina should replace the SCN as a model for circadian clocks, Rosbash (1998) elegantly proposed that fibroblast lines, such as the Rat-1 and NIH/3T3 lines, contain "the vast majority of the molecular machinery that constitutes the mammalian circadian oscillator" and that the study of these mechanisms in these simpler cell cultures will reveal much, if not all, about the arcane workings of the mammalian clock. However, we believe the present study answers that rhetorical question, because despite containing all of the canonical clock genes found within the SCN, fibroblasts are not capable of generating selfsustained circadian rhythms nor of conferring their induced rhythmicity to other cells. Why fibroblasts lack these fundamental circadian properties is presently unclear, although it is possible that all molecular elements of the clock mechanism may not be present or functionally configured or that critical output signals 
found in SCN cells may be absent. Nevertheless, fibroblasts are not pacemakers, whereas the SCN and the SCN2.2 cells are.

With the expected continuum in the intense scientific scrutiny of molecular components of the mammalian biological clock, we believe the model system described here will become an increasingly important tool for the study of circadian pacemaker function. As demonstrated in the present and previous studies (Earnest et al., 1999b), SCN2.2 cells possess both oscillatory and pacemaker properties in vitro, and, critically, these cells are distinguished by the capacity to confer locomotor rhythmicity to arrhythmic, SCN-lesioned rats in vivo. Furthermore, these cells are capable of driving molecular and physiological rhythmicity in $\mathrm{NIH} / 3 \mathrm{~T} 3$ cells via the secretion of an unknown diffusible signal. Obviously, the identity of this signal will provide critical focus for future analysis, but the specificity of this SCN2.2 output also will be of interest. For example, it will be important to determine whether SCN2.2 cells can confer rhythms to neuronal cell lines or even brain tissue explants. So that the physiologically relevant nature(s) of the pacemaker capabilities of SCN2.2 cells in relation to the SCN clock in situ can be addressed further, it will be necessary to determine whether these immortalized cells also can drive circadian rhythms in liver, heart, or endocrine cells. Finally, a comparison of molecular and physiological features in SCN2.2 cells with cells like $\mathrm{NIH} / 3 \mathrm{~T} 3$ fibroblasts or with other tissues, which contain all of the known components of the canonical clockworks (and yet are not in fact clocks), will provide an opportunity to distinguish which unique features of the suprachiasmatic nucleus are necessary for its oscillatory and circadian pacemaking functions as the mammalian circadian clock.

\section{REFERENCES}

Aguilar-Roblero R, Morin LP, Moore RY (1994) Morphological correlates of circadian rhythm restoration induced by transplantation of the suprachiasmatic nucleus in hamsters. Exp Neurol 130:250-260.

Akashi M, Nishida E (2000) Involvement of the MAP kinase cascade in resetting of the mammalian circadian clock. Genes Dev 14:645-649.

Bae K, Jin X, Maywood ES, Hastings MH, Reppert SM, Weaver DR (2001) Differential functions of mPer1, mPer2, and mPer3 in the SCN circadian clock. Neuron 30:525-536.

Balsalobre A, Damiola F, Schibler U (1998) A serum shock induces circadian gene expression in mammalian tissue culture cells. Cell 93:929-937.

Balsalobre A, Brown SA, Marcacci L, Tronche F, Kellendonk C, Reichardt HM, Schutz G, Schibler U (2000) Resetting of circadian time in peripheral tissues by glucocorticoid signaling. Science 289:2344-2347.

Duffield GE, Best JD, Wahleithner JA, Schwartz WJ, Loros JJ, Dunlap JC (2000) Transcriptional profiling of central and peripheral mammalian circadian clocks. Soc Res Biol Rhythms Abstr 7:41.

Earnest DJ, Liang F-Q, DiGiorgio SM, Gallagher MJ, Harvey B, Earnest BJ, Seigel GM (1999a) Establishment and characterization of adenoviral E1A immortalized cell lines derived from the rat suprachiasmatic nucleus. J Neurobiol 39:1-13.

Earnest DJ, Liang F-Q, Ratcliff M, Cassone VM (1999b) Immortal time: circadian clock properties of rat suprachiasmatic cell lines. Science 283:693-695.

Gekakis N, Staknis D, Nguyen HB, Davis FC, Wilsbacher LD, King DP, Takahashi JS, Weitz CJ (1998) Role of the CLOCK protein in the mammalian circadian mechanism. Science 280:1564-1569.

Gillette MU (1997) Regulation of entrainment pathways by the suprachiasmatic circadian clock: sensitivities to second messengers. Prog Brain Res 111:121-132.

Jin X, Shearman LP, Weaver DR, Zylka MJ, De Vries GJ, Reppert SM (1999) A molecular mechanism regulating rhythmic output from the suprachiasmatic circadian clock. Cell 96:57-68.

Klein DC, Moore RY, Reppert SM (1991) Suprachiasmatic nucleus: the mind's clock. New York: Oxford UP.

Kume K, Zylka MJ, Sriram S, Shearman LP, Weaver DR, Jin X, Maywood ES, Hastings MH, Reppert SM (1999) mCry1 and mCry2 are essential components of the negative limb of the circadian clock feedback loop. Cell 98:193-205.

Lehman MN, Silver R, Gladstone WR, Kahn RM, Gibson M, Bittman EL (1987) Circadian rhythmicity restored by neural transplant. Immunocytochemical characterization of the graft and its integration with the host brain. J Neurosci 7:1626-1638.

LeSauter J, Silver R (1998) Output signals of the SCN. Chronobiol Int 15:535-550.

Lopez-Molina L, Conquet F, Dubois-Dauphin M, Schibler U (1997) The DBP gene is expressed according to a circadian rhythm in the suprachiasmatic nucleus and influences circadian behavior. EMBO J 16:6762-6771.

Lowrey PL, Shimomura K, Antoch MP, Yamazaki S, Zemenides PD, Ralph MR, Menaker M, Takahashi JS (2000) Positional syntenic cloning and functional characterization of the mammalian circadian mutation tau. Science 288:483-491.

Newman GC, Hospod FE (1986) Rhythm of suprachiasmatic nucleus 2-deoxyglucose uptake in vitro. Brain Res 381:345-350.

Ralph MR, Menaker M (1988) A mutation of the circadian system in golden hamsters. Science 241:1225-1227.

Ralph MR, Foster RG, Davis FC, Menaker M (1990) Transplanted suprachiasmatic nucleus determines circadian period. Science 247:975-978.

Reppert SM, Weaver DR (2000) Comparing clockworks: mouse versus fly. J Biol Rhythms 15:357-364.

Ripperger JA, Shearman LP, Reppert SM, Schibler U (2000) CLOCK, an essential pacemaker component, controls expression of the circadian transcription factor DBP. Genes Dev 14:679-689.

Rosbash M (1998) Why the rat-1 fibroblast should replace the SCN as the in vitro model of choice. Cell 93:917-919.

Rusak B, Zucker I (1979) Neural regulation of circadian rhythms. Physiol Rev 59:449-526.

Schwartz WJ (1991) SCN metabolic activity in vivo. In: Suprachiasmatic nucleus: the mind's clock (Klein DC, Moore RY, Reppert SM, eds), pp 144-156. New York: Oxford UP.

Shearman LP, Zylka MJ, Weaver DR, Kolakowski Jr LF, Reppert SM (1997) Two period homologs: circadian expression and photic regulation in the suprachiasmatic nuclei. Neuron 19:1261-1269.

Shearman LP, Jin X, Lee C, Reppert SM, Weaver DR (2000a) Targeted disruption of the $\mathrm{mPer} 3$ gene: subtle effects on circadian clock function. Mol Cell Biol 20:6269-6275.

Shearman LP, Sriram S, Weaver DR, Maywood ES, Chaves I, Zheng B, Kume K, Lee CC, van der Horst GTJ, Hastings MH, Reppert SM (2000b) Interacting molecular loops in the mammalian circadian clock. Science 288:1013-1019.

Silver R, LeSauter J, Tresco PA, Lehman MN (1996) A diffusible coupling signal from the transplanted suprachiasmatic nucleus controlling circadian locomotor rhythms. Nature 382:810-813.

Turek FW (1985) Circadian neural rhythms in mammals. Annu Rev Physiol 47:49-64.

van der Horst GTJ, Muijtjens M, Kobayashi K, Takano R, Kanno S, Takao M, de Wit J, Verkerk A, Eker APM, van Leenen D, Buijs R, Bootsma D, Hoeijmakers JHJ, Yasui A (1999) Mammalian Cry 1 and Cry2 are essential for maintenance of circadian rhythms. Nature 398:627-630.

Vitaterna MH, King DP, Chang A-M, Kornhauser JM, Lowrey PL, McDonald JD, Dove WF, Pinto LH, Turek FW, Takahashi JS (1994) Mutagenesis and mapping of a mouse gene, Clock, essential for circadian behavior. Science 264:719-725.

Yagita K, Tamanini F, van der Horst GTJ, Okamura H (2001) Molecular mechanisms of the biological clock in cultured fibroblasts. Science 292:278-281.

Yamazaki S, Numano R, Abe M, Hida A, Takahashi R, Ueda M, Block GD, Sakaki Y, Menaker M, Tei H (2000) Resetting central and peripheral oscillators in transgenic rats. Science 288:682-685.

Young MW (1998) The molecular control of circadian behavioral rhythms and their entrainment in Drosophila. Annu Rev Biochem 67:135-152.

Zheng B, Larkin DW, Albrecht U, Sun ZS, Sage M, Eichele G, Lee CC, Bradley A (1999) The mPer2 gene encodes a functional component of the mammalian circadian clock. Nature 400:169-173.

Zheng BH, Albrecht U, Kaasik K, Sage M, Lu WQ, Vaishnav S, Li Q, Sun ZS, Eichele G, Bradley A, Lee CC (2001) Nonredundant roles of the $\mathrm{mPer} 1$ and $\mathrm{mPer} 2$ genes in the mammalian circadian clock. Cell 105:683-694.

Zylka MJ, Shearman LP, Weaver DR, Reppert SM (1998) Three period homologs in mammals: differential light responses in the suprachiasmatic circadian clock and oscillating transcripts outside of brain. Neuron 20:1103-1110. 